\title{
Musgos (Bryophyta) da Estação Ecológica Wenceslau Guimarães, Estado da Bahia, Brasil ${ }^{1}$
}

\author{
- Milena Evangelista ${ }^{2,4}$, Emilia de Brito Valente ${ }^{2}$, Cid José Passos Bastos ${ }^{3}$ e \\ Silvana B. Vilas Bôas-Bastos ${ }^{3}$
}

Recebido: 1 fevereiro 2019; aceito: 26 agosto 2019

Como citar: Evangelista, M., Valente, E.B., Bastos, C.J.P. e Vilas Bôas-Bastos, S.B. 2019. Musgos (Bryophyta) da Estação Ecológica Wenceslau Guimarães, Estado da Bahia, Brasil. Hoehnea 46: e092019. http://dx.doi.org/10.1590/2236-890609/2019.

\begin{abstract}
Mosses (Bryophyta) from Wenceslau Guimarães Ecological Station, Bahia State, Brazil). In present article, it is presented the floristic composition of mosses (Bryophyta) in a fragment of Montane Atlantic Rainforest in the Wenceslau Guimarães Ecological Station, located in southern Bahia State. As a total, 540 samples were analyzed and 93 species were identified, which are distributed in to 57 genera and 23 families. Trichosteleum cyparissoides is here record for the first time to the Northeast region, and Brittonodoxa lithophila is first referred to Bahia State, both taxa belong to the family Sematophyllaceae.
\end{abstract}

Keywords: diversity, Atlantic Forest, floristic, richness

RESUMO - (Musgos (Bryophyta) da Estação Ecológica Wenceslau Guimarães, Estado da Bahia, Brasil). No presente artigo está sendo apresentado o levantamento florístico dos musgos (Bryophyta) da Estação Ecológica Wenceslau Guimarães, sul do Estado da Bahia, em um fragmento de Floresta Ombrófila Densa montana. Foram analisadas 540 amostras e identificadas 93 espécies, distribuídas em 57 gêneros e 23 famílias. Trichosteleum cyparissoides (Hornsch.) H. Rob tem seu primeiro registro para a região Nordeste e Brittonodoxa lithophila (Hornsch.) W. R. Buck et al. para o Estado da Bahia, ambas pertencentes a família Sematophyllaceae.

Palavras-chave: diversidade, Floresta Atlântica, florística, riqueza

\section{Introdução}

A Estação Ecológica Wenceslau Guimarães representa um importante fragmento de Floresta Ombrófila Densa integrante da zona núcleo da reserva Biosfera da Mata Atlântica reconhecida pela UNESCO. Se caracteriza por ser uma área de relevante interesse ambiental devido à sua diversidade biológica, ocorrência de diversas espécies endêmicas e ameaçadas de extinção e pela importância considerável na proteção dos mananciais hídricos da bacia do Rio das Almas (Decreto No 6.228/1997, Lei $\mathrm{N}^{\mathrm{o}} 7.791 / 2000$, INEMA 2018).
Sua diversidade biológica vem sendo amplamente estudada, e por apresentar uma fisionomia preservada típica de Floresta Ombrófila Densa de formação montana (Veloso et al. 1991) tem fornecido dados importantes para a flora local de angiospermas e contribuições ao projeto Flora da Bahia, com adições ao Estado e descrição de novos táxons para a ciência (Souza 2002, Silva et al. 2003, Rapini \& Farinaccio 2010, Fiaschi \& Amorim 2012, Rigueira et al. 2012, Marinho et al. 2016).

Em seus trabalhos, Reis et al. (2015) investigaram a composição de hepáticas epífitas corticícolas dentro de uma perspectiva ecológica em duas áreas de Floresta

1. Parte da Dissertação de Mestrado do primeiro Autor

2. Universidade Estadual de Feira de Santana, Departamento de Ciências Biológicas, Programa de Pós-Graduação em Botânica, Laboratório de Taxonomia (TAXON), Feira de Santana, 44036-900 Bahia, Brasil

3. Universidade Federal da Bahia, Instituto de Biologia, Laboratório de Taxonomia de Briófitas - BrioFLORA, Campus Ondina, Salvador, 40170-280 Bahia, Brasil

4. Autor para correspondência: milenaevangelista16@gmail.com 
Atlântica na Bahia, dentre elas a Estação Ecológica Wenceslau Guimarães, reportando a ocorrência de espécies endêmicas e o primeiro registro de Frullania macrocephala (Lehm. \& Lindenb.) Lehm. \& Lindenb. para o Brasil. Este se caracterizava até então como o único trabalho sobre briófitas reportado para a Estação Ecológica Wenceslau Guimarães, denotando a importância da realização de inventários mais amplos na área.

Os estudos florísticos com briófitas têm crescido consideravelmente no Estado da Bahia e comprovado a grande representatividade florística existente nas mais diversas fisionomias (Valente \& Pôrto 2006, Vilas Bôas-Bastos \& Bastos 2008a, b, Valente et al. 2009, Vilas Bôas-Bastos \& Bastos 2009, Valente et al. 2011, Oliveira \& Bastos 2014, Vilas Bôas-Bastos et al. 2017). A grande complexidade do Domínio Floresta Atlântica faz com que os inventários florísticos sejam de grande interesse científico. Em função da necessidade em se conhecer com mais detalhes a diversidade e as características das comunidades de briófitas na Estação Ecológica Wenceslau Guimarães, o presente trabalho teve como objetivos, analisar a composição de musgos sob os aspectos florísticos (quanto a riqueza e diversidade) e ecológicos (referentes as comunidades briocenológicas e formas de vida).

\section{Material e métodos}

Área de estudo - A Estação Ecológica Wenceslau Guimarães está situada no distrito de Nova Esperança, sul da Bahia $\left(13^{\circ} 35^{\prime} 43^{\prime \prime} \mathrm{S}\right.$ e $\left.39^{\circ} 43^{\prime} 10^{\prime \prime} \mathrm{W}\right)$, município de Wenceslau Guimarães. Criada e ampliada, respectivamente, pelos Decretos Estaduais $n^{\circ}$ $6.228 / 1997$ e $n^{0} 7.791 / 2000$. Possui uma extensão territorial de 2.418 hectares, relevo montanhoso, altitude variando entre 500-1000 m, clima úmido a sub-úmido, média anual de $18{ }^{\circ} \mathrm{C}$, pluviosidade entre 800-1500 mm com períodos chuvosos prolongados, durante o inverno a região sofre influência da passagem de frentes frias vindas do sul do continente. A Estação Ecológica Wenceslau Guimarães contorna o vale e as cristas que definem o riacho Serra Grande e as cabeceiras do Rio Vermelho (SEAGRI 1997, INEMA 2018). Apresenta importantes fragmentos não contínuos de Floresta Ombrófila Densa e formação de Mata de altitude na encosta do "pico do urubu" (SEAGRI 1997).

Tratamento dos dados - O material foi coletado e herborizado de acordo com a metodologia descrita por Yano (1984). Para a identificação utilizou-se das obras de Florschütz (1964), Manuel (1977, 1977-78), Florschütz-de Waard (1986), Churchill (1988), Allen (1990), Reese (1993), Sharp et al. (1994), Churchill \& Linares (1995), Görts-van Rijn (1996), Buck (1998), Buck (2003), Vaz-Imbassahy et al. (2008) e Bordin \& Yano (2013). O sistema de classificação adotado é o proposto por Goffinet et al. (2009), exceto para a família Sematophyllaceae que foi tratada de acordo com Carvalho-Silva et al. (2017).

As espécies foram classificadas quanto a briocenose, que corresponde ao conjunto de espécies encontradas colonizando determinado tipo de substrato, podendo ser corticícola, epífila, epíxila, terrícola, termitícola e rupícola (Robins 1952, Bates 2009, Vilas Bôas-Bastos et al. 2017). As formas de vida adotadas seguem os conceitos adaptados propostos por Mägdefrau (1982) e Bates (1998), que são consideradas como estratégias para minimizar a evaporação de água e maximizar a produção primária, sendo então classificadas como: tramas, coxins/tufos, dendroides, pendentes e frondes.

Os Domínios Fitogeográficos brasileiros estão de acordo com Fiaschi \& Pirani (2009). Para a distribuição geográfica por Estados brasileiros, foram consultados os catálogos de Costa et al. (2011), Yano (2011), artigos recentemente publicados (e.g. Carmo \& Peralta 2016, Vilas Bôas-Bastos et al. 2017, Carmo et al. 2018) e consultas ao Flora do Brasil (2020, em prep.). A distribuição mundial das espécies foi baseada nos trabalhos de Valente et al. (2009), Vilas Bôas-Bastos \& Bastos (2009), Costa et al. (2011), Oliveira \& Bastos (2014), Carmo \& Peralta (2016) e por consulta à plataforma on line Tropicos ${ }^{\circledR}$.

O cálculo da diversidade das espécies foi realizado com base na relação do número de amostras por espécies analisadas, calculados no software PAST 3.20 (Hammer et al. 2001) a fim de estimar os índices de Shannon (H') e Simpson (1-D). As espécies estão organizadas em ordem alfabética de famílias e gêneros, seguidas das informações sobre distribuição mundial e no Brasil, nos Domínios Fitogeográficos brasileiros, da briocenose, das formas de vida e material selecionado. O material está depositado no Herbário Alexandre Leal Costa (ALCB) do Instituto de Biologia da Universidade Federal da Bahia e as duplicatas no Herbário da Universidade Estadual de Feira de Santana (HUEFS).

\section{Resultados}

Foram identificadas 93 espécies pertencentes a 57 gêneros e 23 famílias (tabela 1). Os musgos 


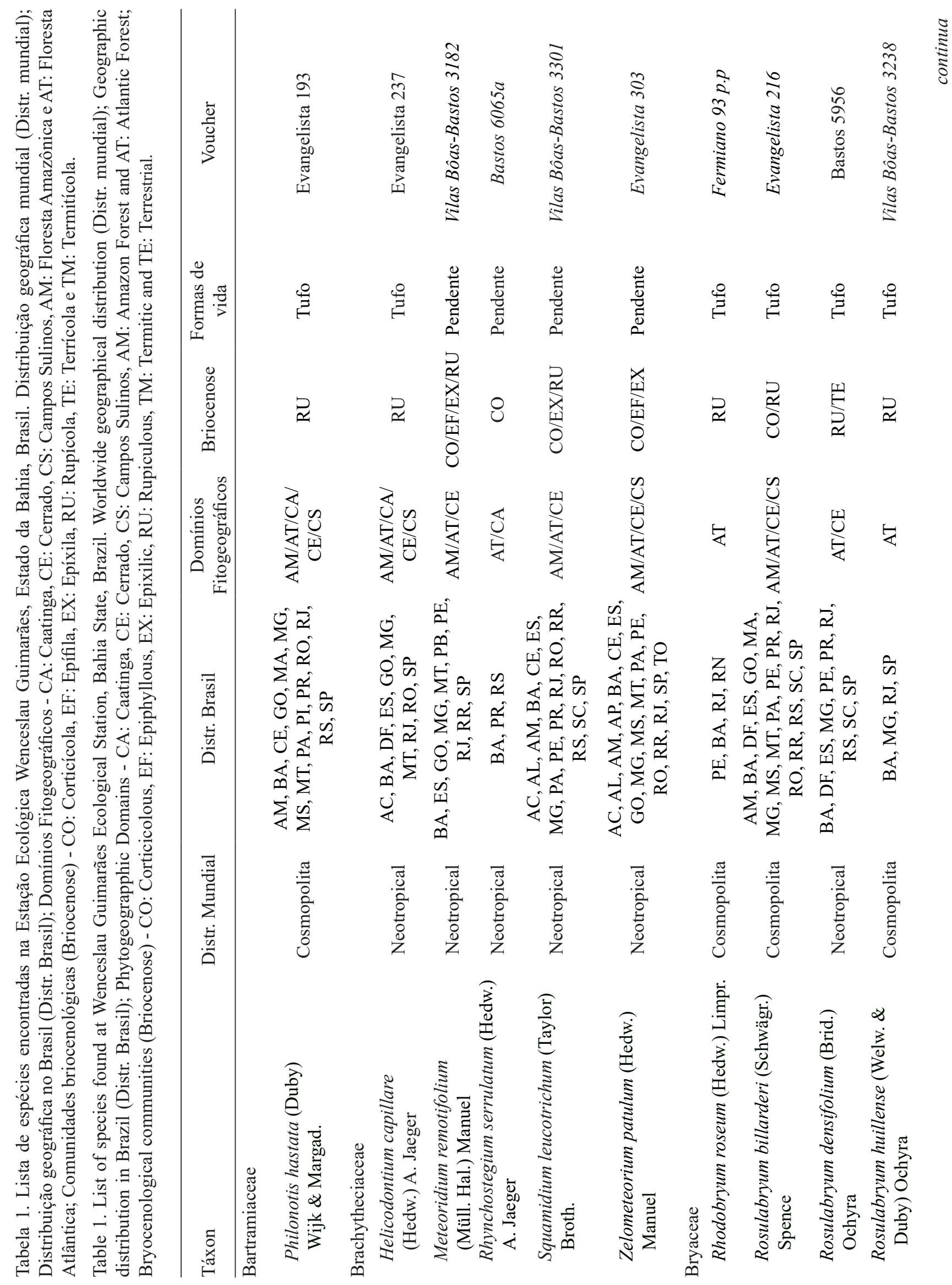




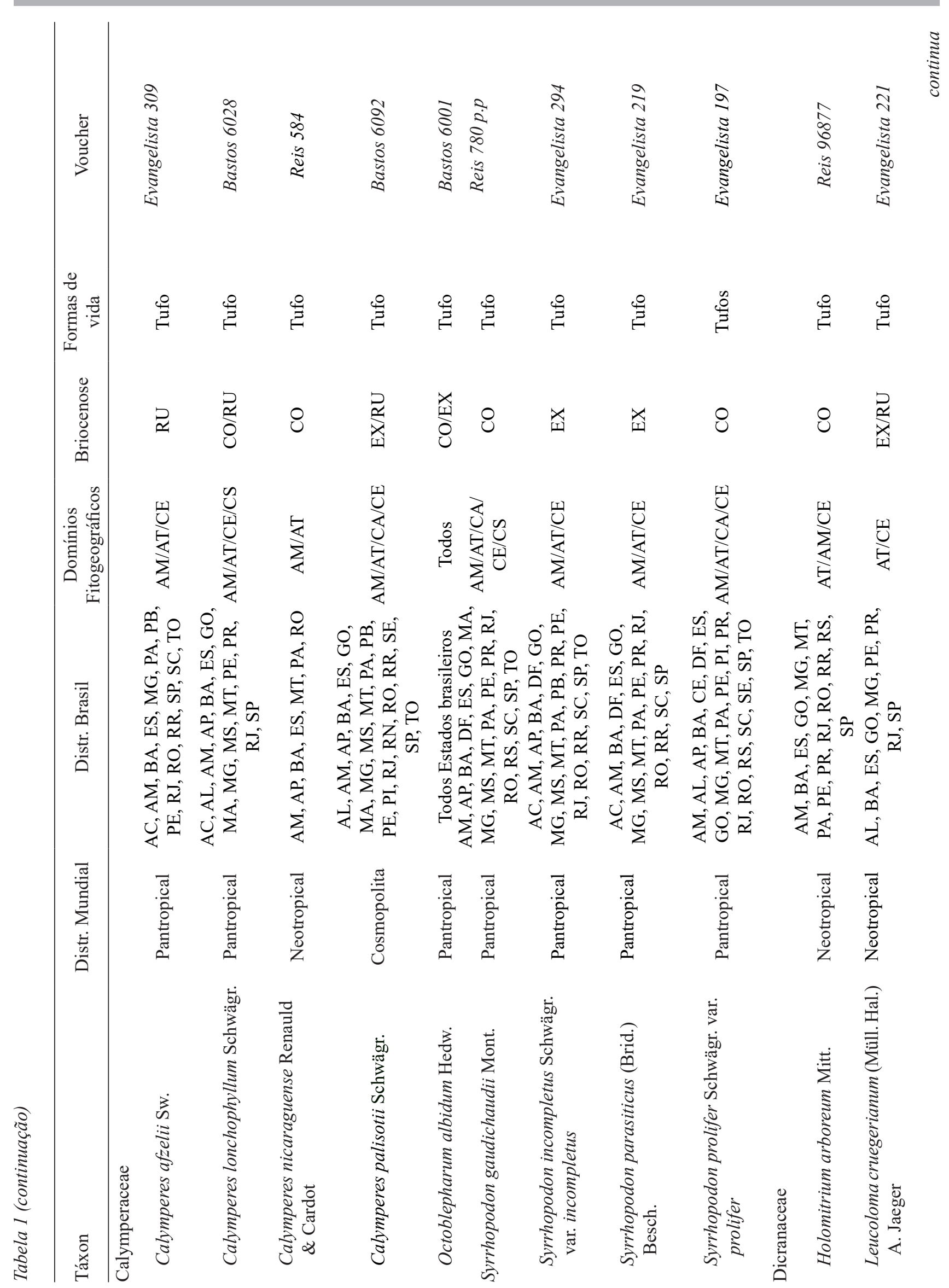




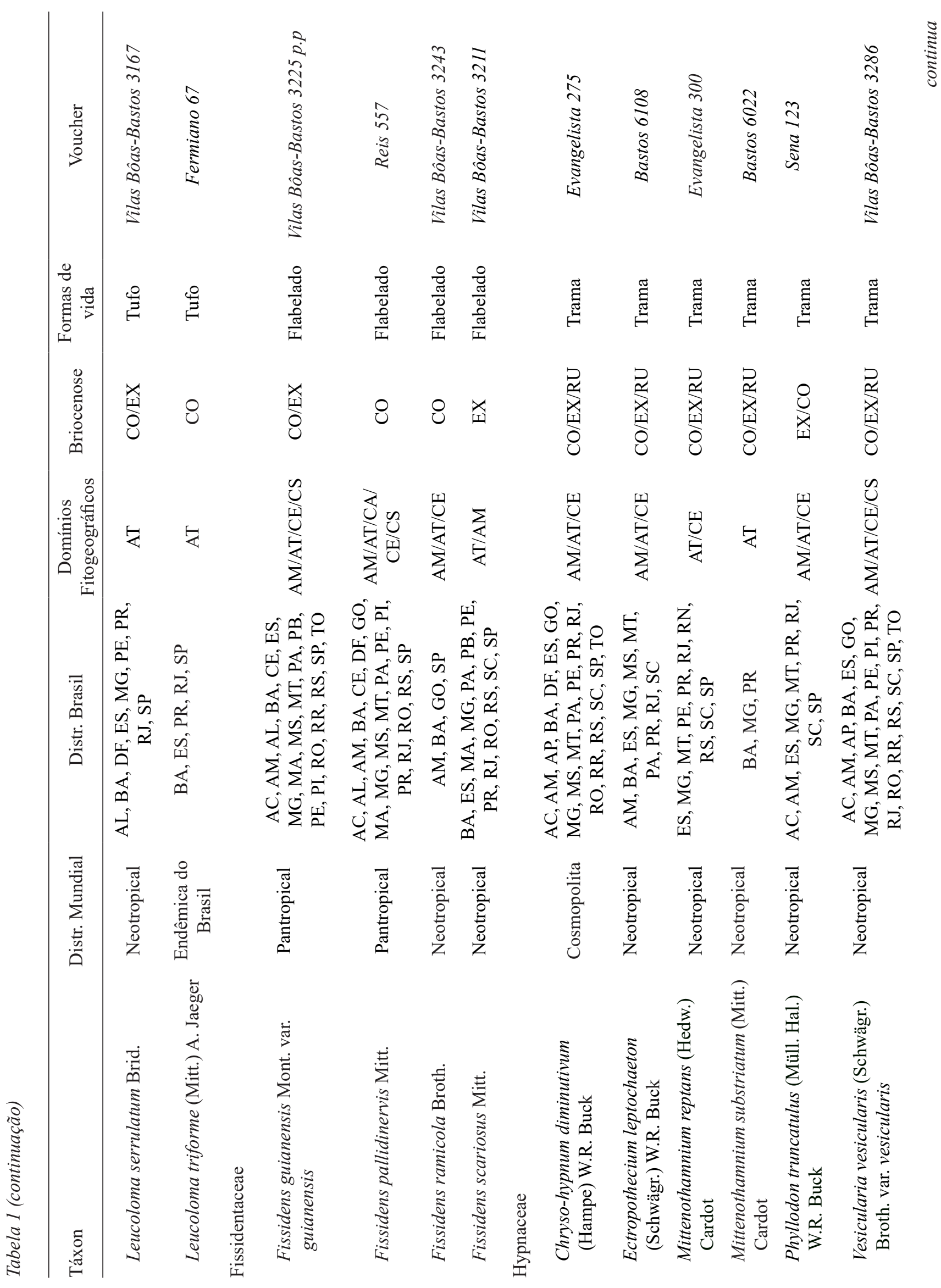




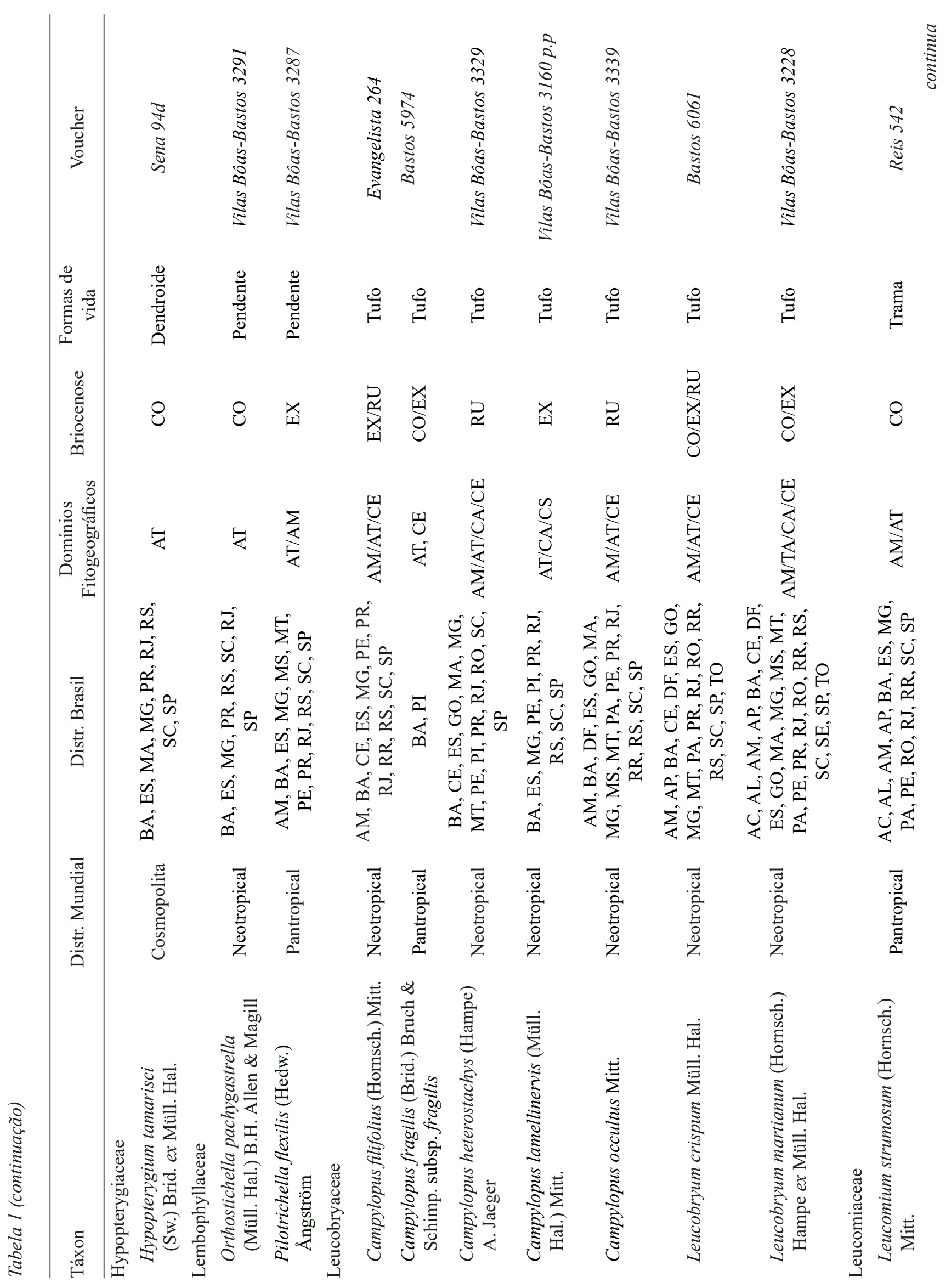




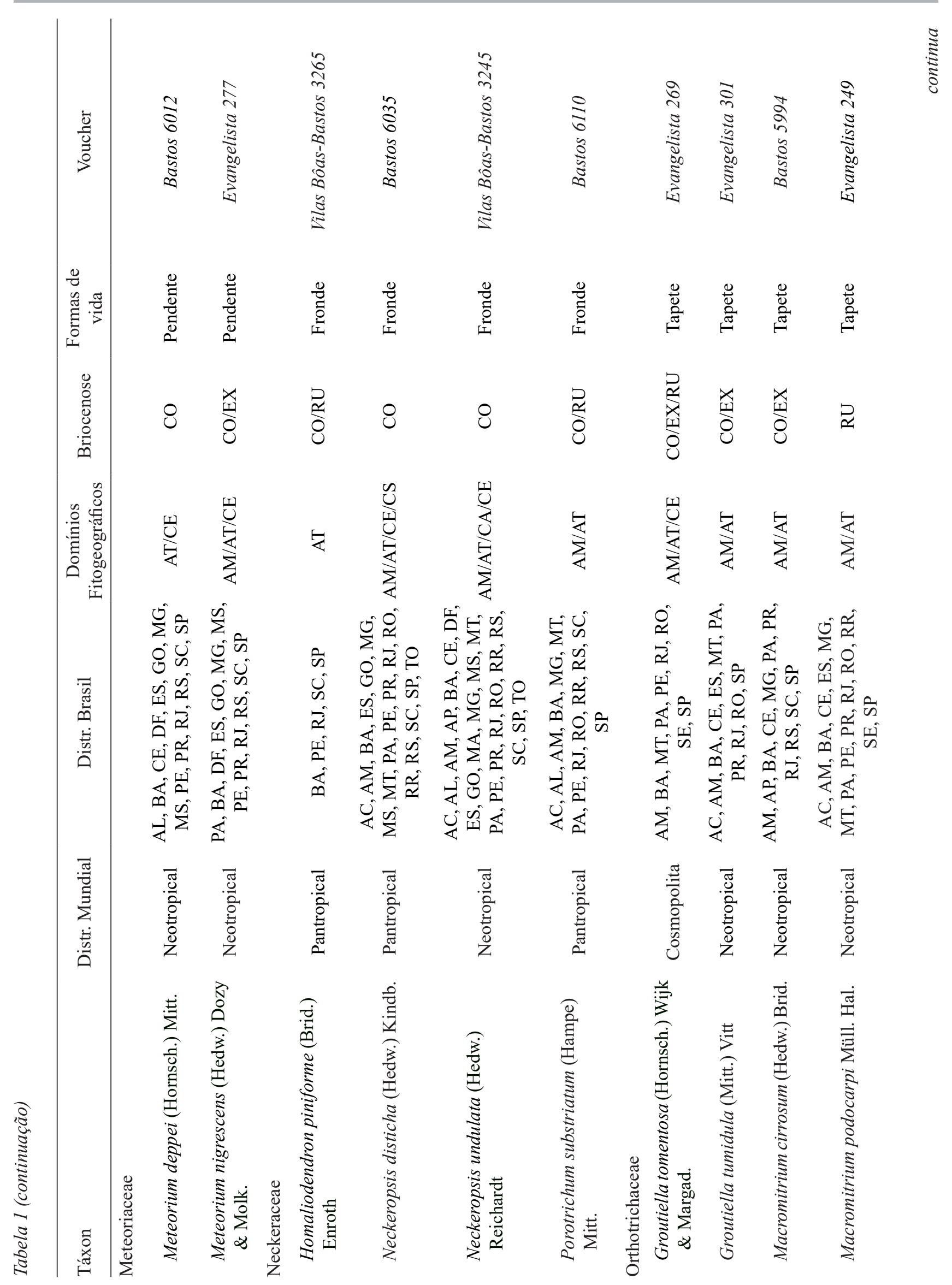




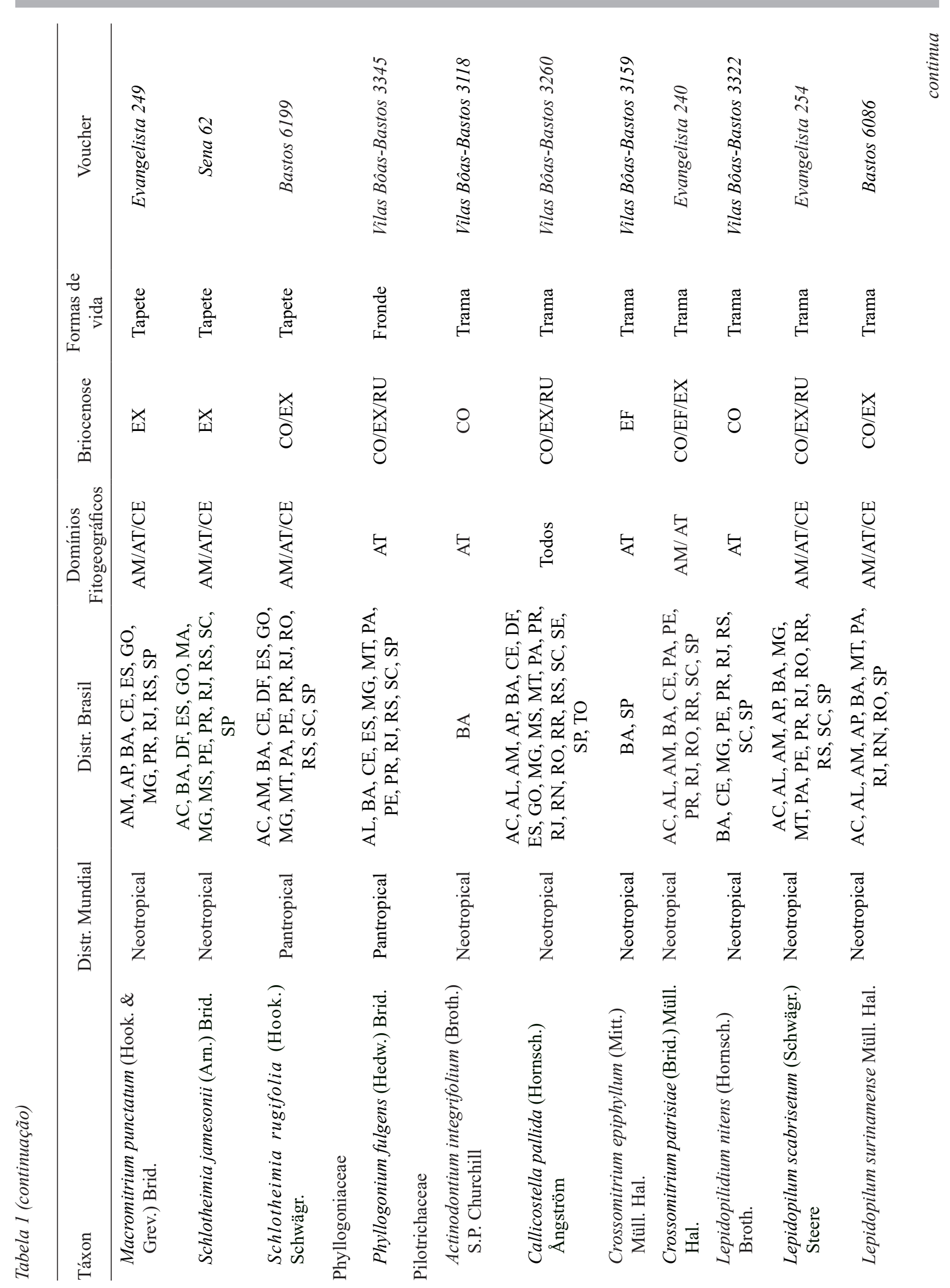




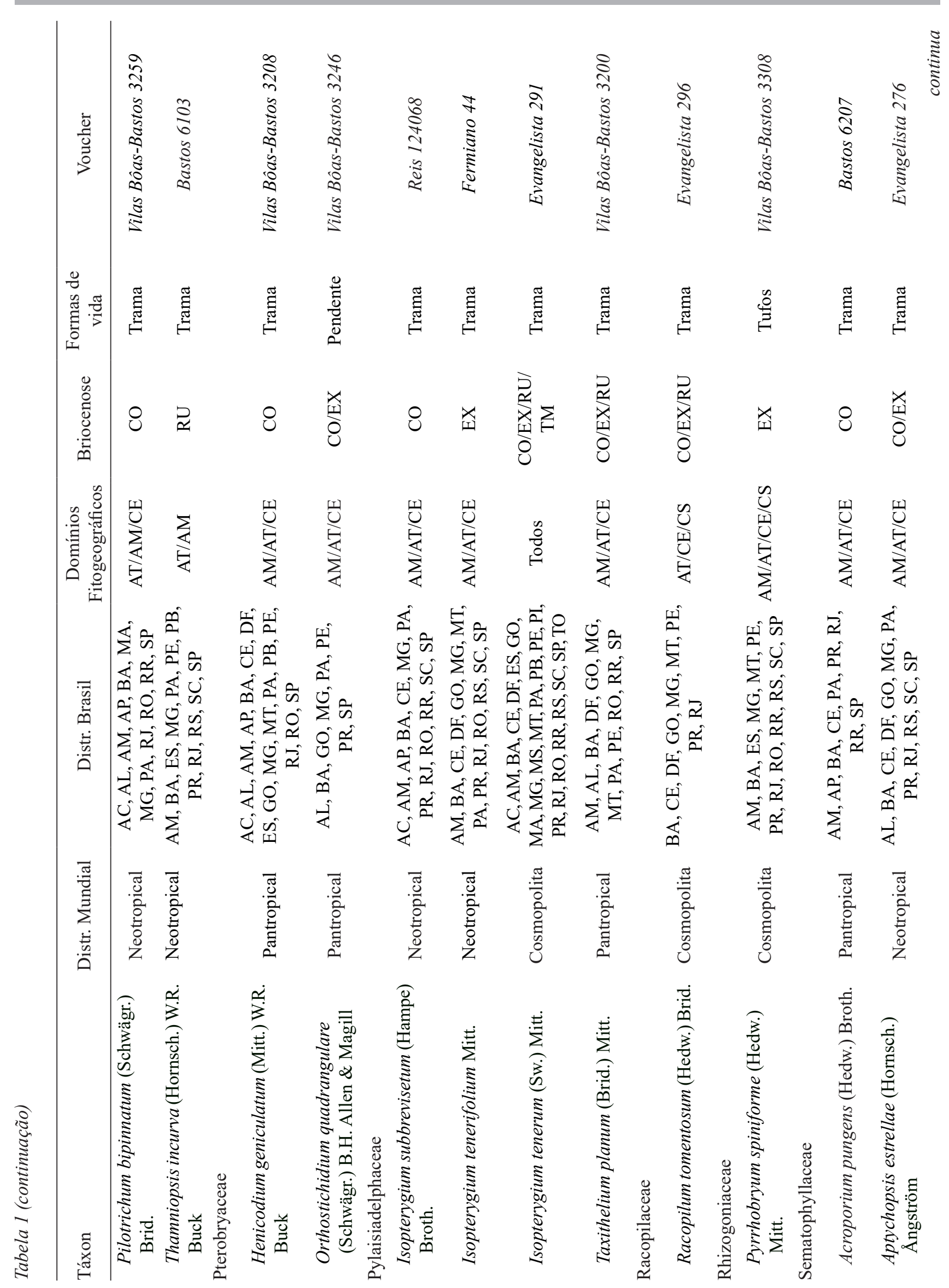




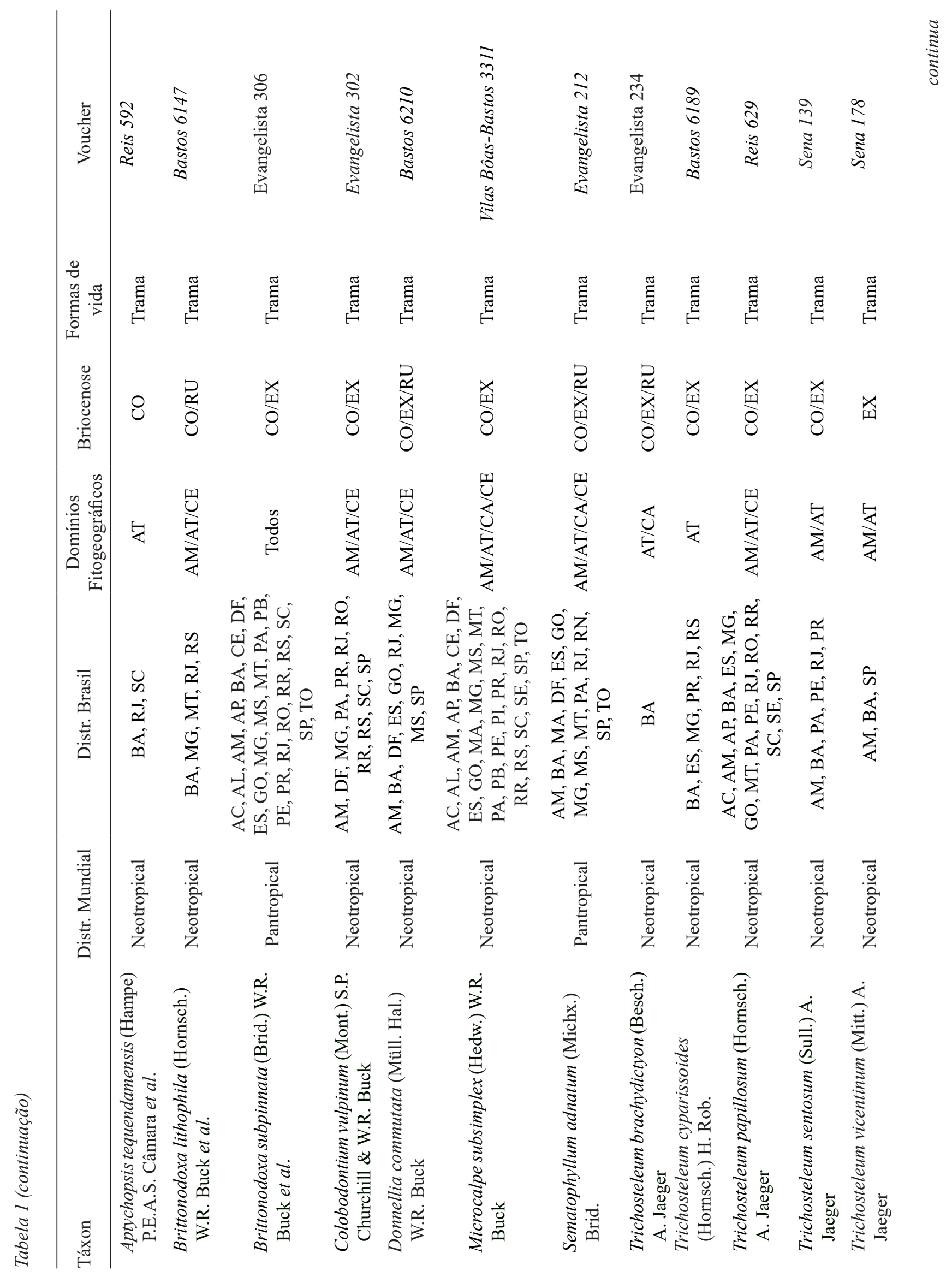


pleurocárpicos (56 espécies) apresentaram maior riqueza em relação aos acrocárpicos (37 espécies). As famílias que apresentaram maior riqueza específica foram Sematophyllaceae (16 espécies), Calymperaceae (nove espécies) e Pilotrichaceae (nove espécies) (figura 1). As famílias que apresentaram maior frequência de ocorrência foram Sematophyllaceae (130 amostras), Calymperaceae (49 amostras), Hypnaceae (48 amostras) e Pilotrichaceae (46 amostras), juntas representam 50,5\% do total das 540 amostras analisadas.

As espécies Sematophyllum adnatum (Michx.) Brid. (37 amostras) e Taxithelium planum (Brid.) Mitt. (29 amostras) apresentaram maior frequência de ocorrência na área. A composição de musgos da Estação Ecológica Wenceslau Guimarães apresentou espécies pouco comuns como Trichosteleum cyparissoides (Hornsch.) H. Rob citada apenas para as regiões Sul e Sudeste; Brittonodoxa lithophila (Hornsch.) W. R. Buck et al. com ocorrência nas regiões Sul e Nordeste apenas para o Estado de Pernambuco.

Em relação a ocorrência das espécies por Domínios Fitogeográficos brasileiros, observou-se que 34 espécies apresentam ocorrência compartilhada entre o Cerrado, Floresta Amazônica e Floresta Atlântica; 12 espécies ocorrem na Floresta Amazônica e Floresta Atlântica; e 14 ocorrem apenas na Floresta Atlântica. As espécies identificadas apresentaram quatro padrões de distribuição geográfica: neotropical (63\%), pantropical (25\%), cosmopolita (11\%) e endêmica do Brasil (Leucoloma triforme (Mitt.) A. Jaeger).

As espécies encontradas na Estação Ecológica Wenceslau Guimarães foram classificadas como corticícola - epíxila - rupícola - epífila - terrícola termitícola (figura 2). Das 540 amostras analisadas, $274(50,7 \%)$ são corticícolas, 166 (30,7\%) epíxilas, $93(17,2 \%)$ rupícolas, quatro $(0,74 \%)$ epífilas, duas $(0,37 \%)$ terrícolas e uma $(0,19 \%)$ termitícola. Das 93 espécies analisadas, 52 são polisubstratos e 41 monosubstratos. As formas de crescimento encontradas em ordem decrescente de ocorrência foram: trama, tufo, pendente, dendroide e fronde (figura 3). Muitas espécies de musgos pleurocárpicos encontrados, apresentam gametófitos dispostos em tramas abertas ou fechadas e foram encontrados colonizando preferencialmente base de árvores vivas e material vegetal em decomposição. Os índices de diversidade de Shannon e Simpson foram, respectivamente $\mathrm{H}^{\prime}=4,078$ e $1-\mathrm{D}=0,98$. 


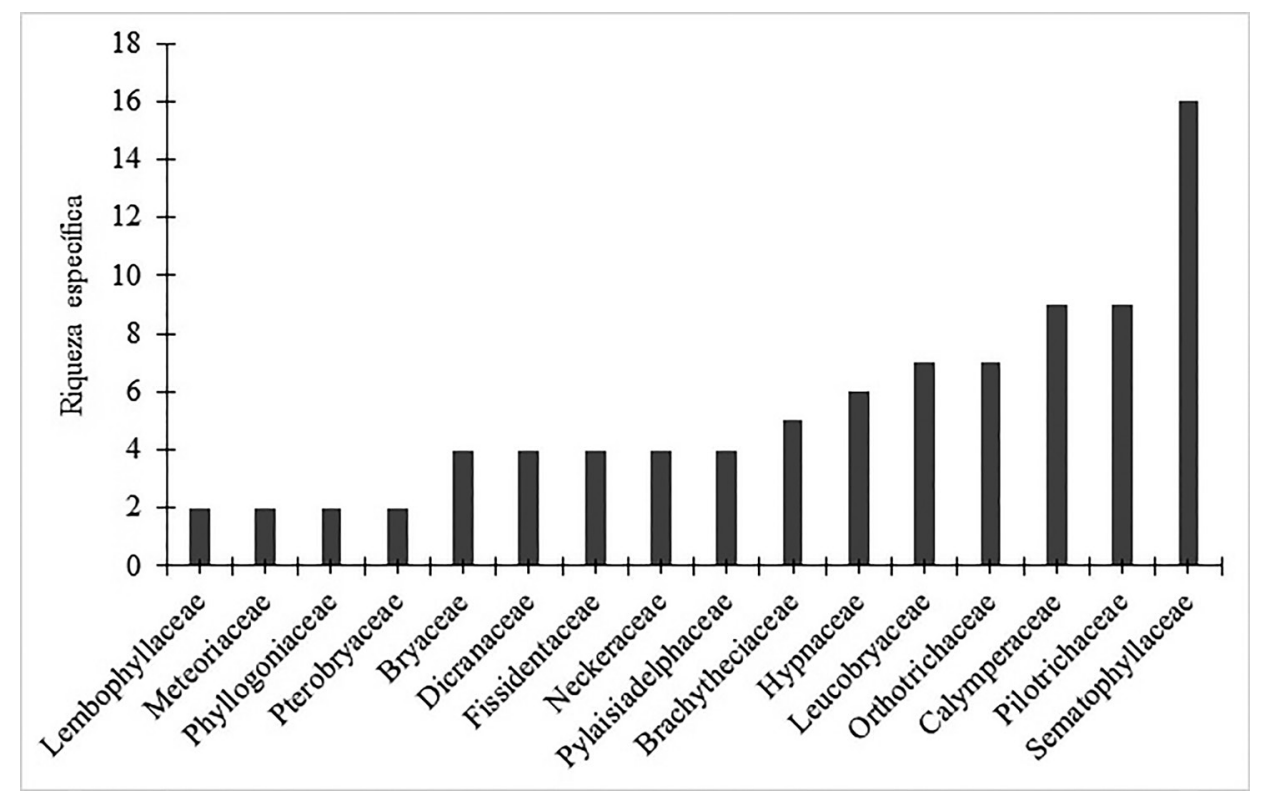

Figura 1. Número de espécies por família de musgos com duas ou mais espécies ocorrentes na Estação Ecológica Wenceslau Guimarães, Estado da Bahia, Brasil.

Figure 1. Number of species per family of mosses with two or more species occurring in the Wenceslau Guimarães Ecological Station, Bahia State, Brazil.

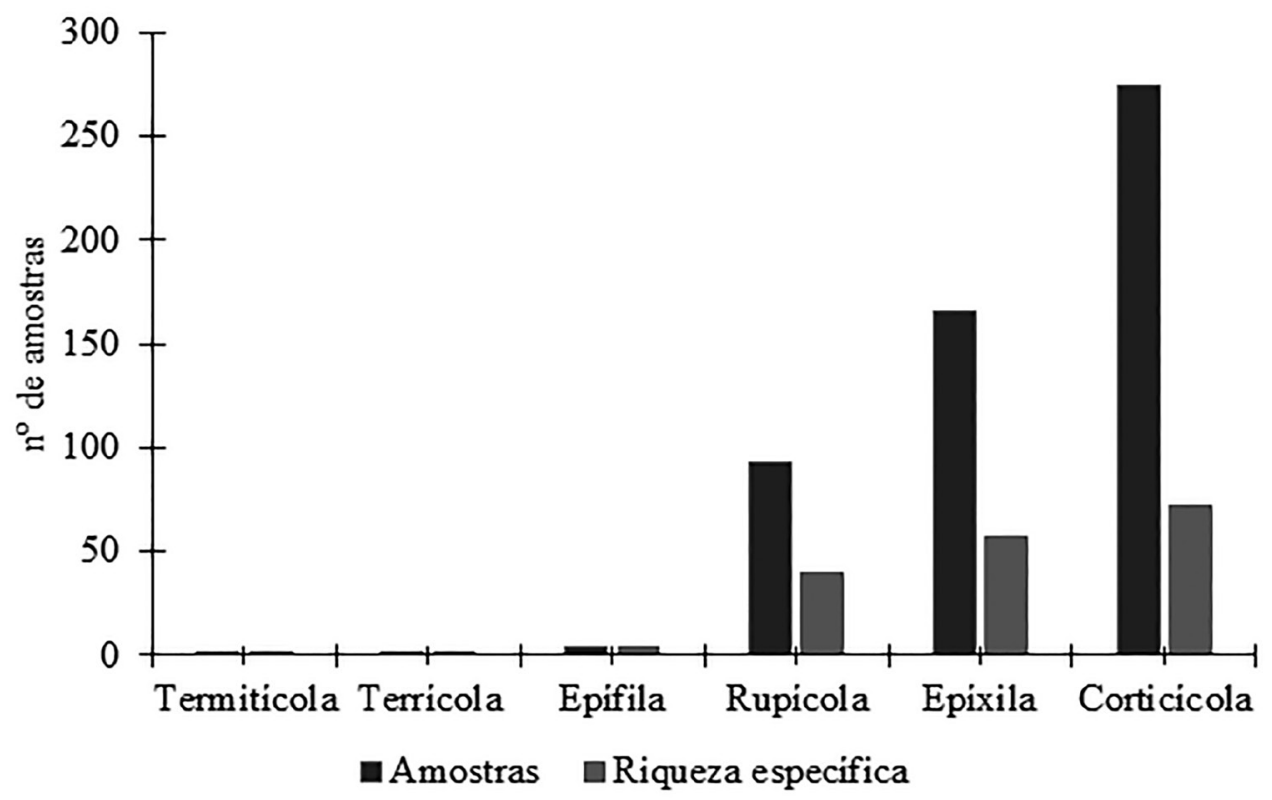

Figura 2: Relação entre a ocorrência de amostras e espécies de acordo com as comunidades briocenológicas encontradas na Estação Ecológica Wenceslau Guimarães, Estado da Bahia, Brasil.

Figure 2. Relationship between the occurrence of samples and species according to the bryocenological communities found at Wenceslau Guimarães Ecological Station, Bahia State, Brazil. 


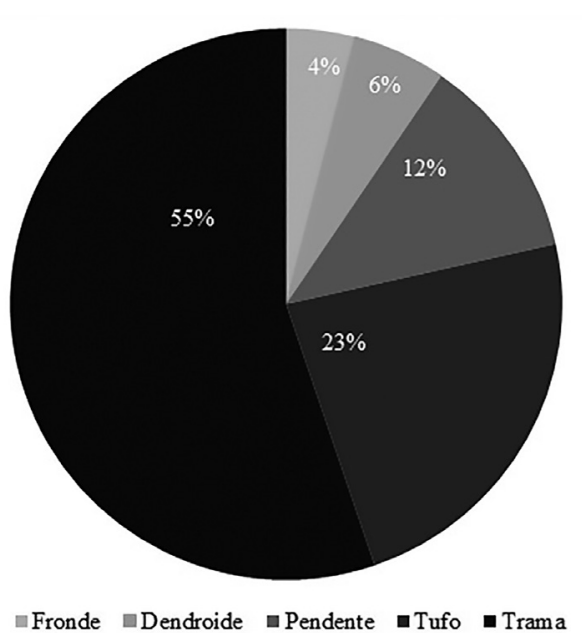

Figura 3: Porcentagem das amostras de acordo com a forma de vida encontrada na Estação Ecológica Wenceslau Guimarães.

Figure 3. Percentage of samples according to life form found at Wenceslau Guimarães Ecological Station, Bahia State, Brazil.

\section{Discussão}

O total de espécies encontradas na Estação Ecológica Wenceslau Guimarães representa 33\% do total de musgos inventariados para o Estado da Bahia (Flora do Brasil 2020, em prep.). Atualmente, são conhecidas 403 espécies de musgos distribuídas dentro do Domínio Fitogeográfico Floresta Atlântica na região Nordeste (Silva \& Pôrto 2014). De acordo com as mesmas autoras, entre os inventários realizados pode-se observar a predominância das famílias Fissidentaceae (45 sp.), Calymperaceae (37 sp.), Leucobryaceae (33 sp.) e Bryaceae (32 sp.), ao contrário dos inventários florísticos realizados no Domínio Floresta Atlântica no Estado da Bahia onde destacaram-se as famílias Sematophyllaceae e Calymperaceae (Valente et al. 2009, Rodrigues \& Valente 2015, Souza et al. 2015, Vilas Bôas-Bastos et al. 2017).

A família Sematophyllaceae está representada por aproximadamente 20 espécies no Estado (Flora do Brasil 2020, em prep.), deste total 14 foram encontradas na Estação Ecológica Wenceslau Guimarães e duas novas ocorrências foram reportadas (Brittonodoxa lithophila e Trichosteleum cyparissoides). Sematophyllaceae está entre as dez maiores famílias de musgos do Brasil (Costa \& Peralta 2015) e possui distribuição pantropical, sendo provavelmente a família de musgo mais comum nos trópicos, com centros de diversidade no Sudeste Asiático e Neotrópico (Carvalho-Silva et al. 2017).
Calymperaceae possui aproximadamente 20 espécies ocorrentes no Estado (Flora do Brasil 2020, em prep.), está entre as dez maiores famílias de musgos ocorrentes no país (Costa \& Peralta 2015) e apresenta distribuição pantropical, ocorrendo preferencialmente em florestas montanas (Edwards 1980).

Algumas espécies de musgos apresentam maior amplitude ecológica ocorrendo em diferentes Domínios Fitogeográficos brasileiros, a exemplo Brittonodoxa subpinnata (Brid.) W.R. Buck et al., Callicostella pallida (Hornsch.) Ångström, Isopterygium tenerum (Sw.) Mitt. e Octoblepharum albidum Hedw. De acordo com Costa \& Peralta (2015), os Domínios Fitogeográficos Cerrado, Floresta Amazônica e Floresta Atlântica apresentam maior diversidade em número de espécies de briófitas. Algumas espécies apresentam ocorrência disjunta entre a Floresta Amazônica e Floresta Atlântica, que pode ser justificado pelo contato que esses Domínios tiveram no passado (Leitão-Filho 1987). A Floresta Atlântica, por sua vez, é considerada um dos principais centros de endemismo, dada as condições ambientais encontradas que favorecem o estabelecimento das briófitas (Costa \& Peralta 2015). As florestas tropicais abrigam os mais elevados índices de diversidade biológica e apresentam maior número de briófitas que qualquer outra região do mundo (Oliveira et al. 2011).

Os inventários florísticos no Estado da Bahia são mais intensificados na Chapada Diamantina e nos fragmentos de Floresta Ombrófila, principalmente aqueles encontrados no Sul do Estado. A composição florística encontrada na Estação Ecológica Wenceslau Guimarães, apresenta algumas espécies comuns aquelas encontradas nos fragmentos de Floresta Ombrófila localizadas no Litoral Norte e Norte do Estado (Vilas Bôas-Bastos \& Bastos 1998, Bastos \& Vilas Bôas-Bastos 2017), nos fragmentos de Floresta Ombrófila Densa montana ou submontana na região Sul do Estado (Vilas Bôas-Bastos \& Bastos 2009, Valente et al. 2009, Souza et al. 2015), assim como na Chapada Diamantina (Valente et al. 2011).

O Litoral Norte apresenta diferentes formações vegetacionais, como Matas Secas, Restingas, Floresta Ombrófila Densa e Florestas Estacionais (Matos et al. 2017), enquanto que aquelas encontradas mais ao Sul do Estado são caracterizadas por formações de Floresta Ombrófila Densa submontana ou montana (Amorim et al. 2009, Rocha \& Amorim 2012). A Chapada Diamantina apresenta diversos tipos vegetacionais como por exemplo os Campos Rupestres, Cerrados de Altitudes e Matas de Altitude (Ribeiro et al. 2005). 
Atrelada aos diferentes tipos vegetacionais existem os fatores abióticos que influenciam na composição florística destes fragmentos. Quanto mais distante do litoral estiver um determinado fragmento, é esperada uma redução das precipitações e, consequentemente dos níveis de umidade (Matos et al. 2017), dificultando o estabelecimento de algumas espécies de briófitas.

Trichosteleum cyparissoides está associada a elevadas altitudes sendo reportada apenas para o Domínio Floresta Atlântica em áreas serranas ou montanas (Costa \& Lima 2005, Peralta et al. 2008, em ambos como Sematophyllum cyparissoides (Hornsch.) R.S. Williams). Brittonodoxa lithophila é reportada para áreas de altitudes em Floresta Ombrófila Densa Montana e Alto Montana (Carmo et al. 2016 como Sematophyllum lithophilum (Hornsch.) Ångstr.), apresenta distribuição mais acentuada no Sul do país, para a região Nordeste é citada apenas para o Estado de Pernambuco (Costa et al. 2011, Yano $2011 \mathrm{em}$ ambos como Sematophyllum lithophilum).

Algumas espécies pouco comuns no país, foram reportadas para a Estação Ecológica Wenceslau Guimarães. Campylopus fragilis subsp. fragilis (Leucobryaceae) apresenta ocorrência no Brasil apenas para os Estados do Piauí e Bahia, neste último ocorrendo na Chapada Diamantina, em Campo Rupestre (Valente et al. 2011), sendo então a primeira referência dessa subespécie para Floresta Ombrófila Densa. Leucoloma triforme (Dicranaceae) é uma espécie endêmica do Brasil, tem ocorrência no Centro Sul, preferencialmente em fragmentos de Floresta Ombrófila (Gama et al. 2015).

As briófitas, de forma geral são preferencialmente encontradas colonizando troncos de árvores vivas, uma vez que eles proporcionam condições favoráveis para o seu estabelecimento (Gradstein \& Pócs 1989), com o pH ácido e córtex rugoso auxiliando na retenção de água e nutrientes (Frahm 1990). De acordo com Bates (1998), as formas de vida estão associadas com adaptações para maximização e eficiência no uso da água, intensidade luminosa e substrato. Os musgos que apresentam forma de vida do tipo trama, apresentam gametófitos dispostos de forma a maximizar a captura de luz (Bates 1998).

O índice de diversidade encontrado $\left(\mathrm{H}^{\prime}=4,078\right)$ é similar àqueles encontrados em outros inventários florísticos com briófitas em Florestas Ombrófilas (e.g. Oliveira-e-Silva et al. 2002, Santos et al. 2011). Os dados encontrados corroboram o fato sobre os fragmentos de Floresta Ombrófila apresentarem os mais elevados níveis de riqueza e diversidade vegetal (Kakashima et al. 2015). Dado o valor da equabilidade encontrado $(1-\mathrm{D}=0,98)$ pode-se inferir que as espécies encontradas apresentaram distribuição uniforme dentro da comunidade com pouca dominância acentuada de qualquer espécie, resultados parecidos foram encontrados por Campelo \& Pôrto (2007).

\section{Agradecimentos}

Os autores agradecem à Universidade Federal da Bahia pelo suporte logístico para as coletas. A Coordenação de Aperfeiçoamento de Pessoal de Nível Superior - Brasil (CAPES) - Código de financiamento 001, com apoio nas passagens e estadias ao primeiro autor. Fundação de Amparo à Pesquisa do Estado da Bahia (FAPESB) pela bolsa concedida ao primeiro autor. Instituto de Meio Ambiente e Recursos Hídricos (INEMA) pela autorização cedida para a realização de coletas na Estação Ecológica Wenceslau Guimarães (Portaria $\mathrm{n}^{\circ} 9.095$ de 15 de janeiro de 2015 e Portaria $\mathrm{n}^{\mathrm{o}} 15.191$ de 09 de novembro de 2017). À Dr. ${ }^{\mathrm{a}}$ Juçara Bordin, pela confirmação de Fissidens ramicola Broth. e aos estagiários do Laboratório de Taxonomia de Briófitas (BrioFLORA) pelo auxilio nas coletas, triagem e herborização do material.

\section{Literatura citada}

Allen, B.H. 1990. A revision of the genus Crossomitrium (Musci: Hookeriaceae). Tropical Bryology 2: 3-32.

Amorim A.M., Jardim, J.G., Lopes, M.M.M., Fiaschi, P., Borges, R.A.X., Perdiz, R.O \& Thomas, W.W. 2009. Angiospermas em remanescentes de floresta montana no sul da Bahia, Brasil. Biota Neotropica 9: 313-348.

Bastos, C.J.P., Yano, O. \& Vilas Bôas-Bastos, S.B. 2000. Briófitas de campos rupestres da Chapada Diamantina, Estado da Bahia, Brasil. Revista Brasileira de Botânica 23: 359-370.

Bastos, C.J.P. \& Vilas Bôas-Bastos, S.B. 2017. Diversidade florística e espectro ecológico das briófitas. In: J.M.C. Nunes \& M.R.B. Matos (eds.). Litoral Norte da Bahia: Caracterização Ambiental, Biodiversidade e Conservação. 1 ed. Editora da Universidade Federal da Bahia, Salvador, pp. 117-127.

Bates, J.W. 1998. Is 'Life-Form' a useful concept in bryophyte ecology? Nordic Society Oikos 82: 223-237.

Bates, J.W. 2009. Mineral nutrition and substratum ecology. In: B. Goffinet \& A.J. Shaw. Bryophyte Biology. Cambridge University Press, Cambridge, pp. 299-356. 
Bordin, J. \& Yano, O. 2013. Fissidentaceae (Bryophyta) do Brasil. Boletim do Instituto de Botânica 22: 1-72.

Buck, W.R. 1998. Pleurocarpous mosses of the West Indies. Memoirs of the New York Botanical Garden 1: 1-401.

Buck, W.R. 2003. Guide to the plants of Central French Guiana. Part 3. Mosses. Memoirs of the New York Botanical Garden 76: 1-167.

Carmo, D.M. \& Peralta, D.F. 2016. Survey of bryophytes in Serra da Canastra National Park, Minas Gerais, Brazil. Acta Botanica Brasilica 30: 462-472.

Carmo, D.M., Lima, J.S., Amélio L.A. \& Peralta, D.F. 2016. Briófitas do Parque Estadual da Serra do Mar, Núcleo de Santa Virgínia, Estado de São Paulo, Brasil. Hoehnea 42: 265-287.

Carmo, D.M., Lima, J.S., Silva, M.I., Amélio, L.A. \& Peralta, D.F. 2018. Briófitas da reserva particular do Patrimônio Natural da Serra do Caraça, Estado de Minas Gerais, Brasil. Hoehnea 45: 1-25.

Campelo, M.J.A. \& Pôrto, K.C. 2007. Riqueza e composição de briófitos epífitos em fanerógamas, Pernambuco, Brasil. Revista Brasileira de Biociências 5: 621-623.

Carvalho-Silva, M., Stech, M., Soares-Silva, L.H., Buck, W.R., Wickett, N.J., Liu, Y \& Câmara, P.E.A.S. 2017. A molecular phylogeny of the Sematophyllaceae s.l. (Hypnales) based on plastid, mitochondrial and nuclear markers, and its taxonomic implications. Taxon 66: 811-831.

Churchill, S.P. 1988. A revision of the moss genus Lepidopilum (Callicostaceae). Dissertação de Mestrado, Universidade de Nova York, Nova York.

Churchill, S.P. \& Linares, C.E. 1995. Prodromus Bryologiae Novo-Granatensis: Introduccion a la Flora de Musgos de Colombia. Biblioteca José Jerónimo Triana 12: 1-924.

Costa, D.P., Pôrto, K.C., Luizi-Ponzo, A.P., Ilkiu-Borges, A.L., Bastos, C.J.P., Câmara, P.E.A.S., Peralta, D.F., Bôas-Bastos, S.B.V., Imbassahy, C.A.A., Henriques, D.K., Gomes, H.C.S., Rocha, L.M., Santos, N.D., Siviero, T.S., Vaz-Imbassahy, T.F. \& Churchill, S.P. 2011. Synopsis of the Brazilian moss flora: checklist, distribution and conservation. Nova Hedwigia 93: 277-334.

Costa, D.P. \& Lima, F.M. 2005. Moss diversity in the tropical rainforests of Rio de Janeiro, southeastern Brazil. Revista brasileira de botânica 28: 671-685.

Costa, D.P. \& Peralta, D.F. 2015. Bryophytes diversity in Brazil. Rodriguésia 66: 1063-1071.

Edwards, R. 1980. A revision of West Tropical African Calymperaceae I. Introduction and Calymperes. Journal of Bryology 11: 49-93.

Fiaschi, P \& Pirani, J.R. 2009. Review of plant biogeographic studies in Brazil. Journal of Systematics and Evolution 47: 477-496.
Fiaschi, P. \& Amorim, A.M. 2012. Flora da Bahia: Dichapetalaceae. Sitientibus série Ciências Biológicas 12: 201-206.

Flora do Brasil 2020. 2019. Jardim Botânico do Rio de Janeiro. Disponível em http://floradobrasil.jbrj.gov.br (acesso em 30-IV-2019).

Florschütz, P.A. 1964. The moss of Suriname - Part I. Leiden, E.J. Brill.

Florschütz-de Waard, J. 1986. Flora of Suriname - Musci: Part II. Leiden, E.J. Brill.

Fhahm, J.P. 1990. The effect of light and temperature on the Growth of the Bryophytes of Tropical forest. Nova Hedwigia 51: 151-164.

Gama, R., Stech, M., Schafer-Verwimp, A. \& Peralta, D.P. 2015. A morpho-molecular revision of Leucoloma (Dicranaceae, Bryophyta) in Brazil. Nova Hedwigia 100: 319-332.

Gradstein, S.R. \& Pócs, T. 1989. Bryophytes. In: H. Lieth \& M.J.A Wagner (eds.). Tropical rain Forest Ecossystems. Elsevier Science Publishers, Amsterdam, pp. 311-315.

Goffinet, B., Buck, W.R. \& Shaw, A.J. 2009. Morphology, anatomy and classification of the Bryophyta. In: B. Goffinet \& A.J. Shaw (eds.). Bryophyte Biology. Cambridge University Press, Cambridge, pp. 55-138.

Görts-van Rijn, A.R.A. 1996. Flora of the Guianas. Series C: Bryophytes. Musci III. The Royal Botanic Gardens, Kew, pp. 363-489.

Hammer, O., Harper, D.A.T. \& Ryan, P.D. 2001. PAST: Paleontological Statistics software package for education and data analysis. Palaentologia Electronica 4: 1-9.

INEMA (Instituto do Meio Ambiente e Recursos Hídricos). 2016. Estação ecológica de Wenceslau Guimarães. Instituto do Meio Ambiente e Recursos Hídricos. Disponível em http://www.inema.ba.gov.br/ gestao-2/unidades-de-conservacao/estacao-ecologica/ estacao-ecologica-de-wenceslau-guimaraes/ (acesso em 25-X- 2017).

Kakashima, S., Morita, S., Yoshida, K., Ishida, A., Hayashi, S., Asami, T., Ito, H., Miller, D.G., Uehara, T., Mori, S., Hasegawa, E., Matsuura, K., Kasuya, E. \& Yoshimura, J. 2015. The contribution of seed dispersers to tree species diversity in tropical rainforests. Royal Society Open Science 2: 1-11.

Leitão-Filho, H.F. 1987. Considerações sobre a florística de florestas tropicais e sub-tropicais do Brasil. Instituto de Pesquisas e Estudos Florestais (IPEF) 35: 41-46.

Mägdefrau, K. 1982. Life-forms of bryophytes. In: A.J.E. Smith (ed.). Bryophyte ecology. Chapman \& Hall, New York, pp. 45-58.

Manuel, M.G. 1977. A monograph of the genus Zelometeorium Manuel, gen. nov. (Bryopsida: Meteoriaceae). The Journal of the Hattori Botanical Laboratory 43: 107-126. 
Manuel, M.G. 1977-1978. The genus Meteoridium (Müll. Hal.) Manuel, stat. nov. (Bryopsida: Meteoriaceae). Lindbergia 4: 45-55.

Marinho, L.C., Carneiro-Torres, D.S., Amorim, A.M., Oliveira, R.J. \& Giulietti, A.M. 2016. Flora da Bahia: Podocarpaceae. Sitientibus, série Ciências Biológicas 16: $1-5$.

Matos, M.R.B., Queiroz, E.P. \& Bautista, H.P. 2017. Fitossociologia. In: J.M.C. Nunes \& M.R.B. Matos (eds.). Litoral Norte da Bahia caracterização ambiental, biodiversidade e conservação. 1 ed. Editora da Universidade Federal da Bahia, Salvador, pp. 1-460.

Oliveira, H.C. \& Bastos, C.J.P. 2014. Briófitas epífitas de fragmentos de Floresta Atlântica da reserva Ecológica Michelin, Estado da Bahia, Brasil. Hoehnea 41: 631-646.

Oliveira, J.R.P.M., Pôrto, K.C. \& Silva, M.P.P. 2011. Richness preservation in a fragmented landscape: A study of epiphytic bryophytes in an Atlantic forest remnant in Northeast Brazil. Journal of Bryology 33: 279-290.

Oliveira-e-Silva, M.I.M.N., Milanez, A.I. \& Yano. O. 2002. Aspectos ecológicos de briófitas em áreas preservadas de mata Atlântica, Rio de Janeiro, Brasil. Tropical Bryology 22: 77-102.

Peralta, D.F., Bordin, J. \& Yano, O. 2008. Novas ocorrências de briófitas nos Estados brasileiros. Hoehnea 35: 123-158.

Presidência da República. 1997. Decreto lei nº.228, de 21-II-1997. Cria a Estação Ecológica Estadual de Wenceslau Guimarães e dá outras providências. Diário Oficial do Estado, Poder Executivo, Bahia, 21-II-1997. Seção I.

Presidência da República. 2000. Decreto lei n 7.791, de 19-IV-2000. Amplia a Estação Ecológica Estadual de Wenceslau Guimarães, alterando dispositivos do Decreto $\mathrm{n}^{\circ}$ 6.228, de 21 de fevereiro de 1997 e dá outras providências. Diário Oficial do Estado, Poder Executivo, Bahia, 19-IV-2000. Seção I.

Rapini, A. \& Farinaccio, M.A. 2010. Oxypetalum laciniatum, uma nova espécie de Asclepiadoideae (Apocynaceae) do sul da Bahia, Brasil. Rodriguésia 61: 17-20.

Reese, W.D. 1993. Calymperaceae. Flora Neotropica Monograph 58:1-102.

Reis, L.C., Oliveira, H.C. \& Bastos, C.J.B. 2015. Hepáticas (Marchantiophyta) epífitas de duas áreas de Floresta Atlântica no Estado da Bahia, Brasil. Pesquisas, Botânica 67: 225-241.

Ribeiro, P.L., Borba, E.L. \& Toscano-de-Brito, A.L.V. 2005. O gênero Bulbophyllum Thouars (Orchidaceae) na Chapada Diamantina, Bahia, Brasil. Revista Brasileira de Botânica 28: 423-439.
Rigueira, D.M.C., Molinari, A.L.M.M., Mariano, D.L.S., Reis, R.M., Portugal, A.B., Santana, N.S. \& Santos, R.A. 2012. Influência da distância da borda e do adensamento foliar sobre a abundância de plantas pioneiras em um fragmento de floresta tropical sobmontana na Estação Ecológica de Wenceslau Guimarães (Bahia, Brasil). Acta Botanica Brasilica 26: 197-202.

Robins, R.G. 1952. Bryophyte ecology of a dune area in New Zealand. Acta Geobotanica 4: 1-31.

Rocha, D.S.B. \& Amorim, A.M.A. 2012. Heterogeneidade altitudinal na Floresta Atlântica setentrional: um estudo de caso no sul da Bahia, Brasil. Acta Botanica Brasilica 26: 309-327.

Rodrigues, A.F.A.S. \& Valente, E.B. 2015. Diversidade de musgos (Bryophyta) de um fragmento de floresta ombrófila no município de Barra do Choça, Bahia. Pesquisas, Botânica 67: 143-148.

Santos, N.D., Costa, D.P., Kinoshita, L.S. \& Sheperd, G.J. 2011. Aspectos brioflorísticos e fitogeográficos de duas formações costeiras de Floresta Atlântica da Serra do Mar, Ubatuba/SP, Brasil. Biota Neotropica 11: 425-438.

SEAGRI (Secretaria da Agricultura, Pecuária, Irrigação, Pesca e Aquicultura). 1997. Plano de Manejo Estação Ecológica Estadual Wenceslau Guimarães. Secretaria da Agricultura, Pecuária, Irrigação, Pesca e Aquicultura, Salvador.

Sharp, A.J., Crum, H. \& Eckel, P.M. 1994. The moss flora of Mexico. Memoirs of The New York Botanical Garden 69: 1-1113.

Silva, S.N., Carvalho, A.M.V. \& Santos, F.A.R. 2003. Cestrum L. (Solanaceae) da Mata Higrófila do Estado da Bahia, Brasil. Acta Scientiarum: Biological Sciences 25: 157-166.

Silva, M.P.P. \& Pôrto, K.C. 2014. Briófitas: estado do conhecimento e vulnerabilidade na Floresta Atlântica Nordestina. Boletim do Museu de Biologia Mello Leitão 36: 19-34.

Souza, M.L.D.R. 2002. O gênero Ossea DC. (Melastomataceae) no Brasil: circunscrição e notas taxonômicas. Insula 31: 1-28.

Souza, A.M., Valente, E.B. \& Azevedo, C.O. 2015. Musgos de um fragmento de floresta estacional semidecidual do município de Vitória da Conquista, Bahia, Brasil. Pesquisas, Botânica 67: 217-233.

Valente, E.B. \& Pôrto, K.C. 2006. Hepáticas (Marchantiophyta) de um fragmento de Mata Atlântica na Serra da Jiboia, município de Santa Teresinha, BA, Brasil. Acta Botanica Brasilica 20: 433-441.

Valente, E.B., Pôrto, K.C., Vilas Bôas-Bastos, S.B. \& Bastos, C.J.P. 2009. Musgos (Bryophyta) de um fragmento de Mata Atlântica na Serra da Jiboia, município de Santa Terezinha, BA, Brasil. Acta Botanica Brasilica 23: 369-375. 
Valente, E.B., Pôrto, K.C. \& Bastos, C.J.P. 2011. Check list of Bryophytes of Chapada Diamantina, Bahia. Boletim do Instituto de Botânica 21: 111-124.

Vaz-Imbassahy, T.F., Imbassay, C.A.A. \& Costa, D.P. 2008. Sinopse de Pilotrichaceae (Bryophyta) no Brasil. Rodriguésia 59: 765-797.

Veloso, H.P., Rangel Filho, A.L. \& Lima, J.C.A. 1991. Classificação da vegetação brasileira, adaptada a um sistema universal. IBGE, Rio de Janeiro, pp. 124.

Vilas Bôas-Bastos, S.B. \& Bastos, C.J.P. 1998. Adições a brioflora (Bryopsida) do Estado da Bahia, Brasil. Tropical Bryology 15: 111-116.

Vilas Bôas-Bastos, S.B. \& Bastos, C.J.P. 2008a. Neckeraceae Schimp. (Bryophyta, Bryopsida) da Reserva Ecológica de Michelin, Município de Igrapiúna, Bahia, Brasil. Sitientibus série Ciências Biológicas 8: 263-274.

Vilas Bôas-Bastos, S.B. \& Bastos, C.J.P. 2008b. Musgos acrocárpicos e cladocárpicos (Bryophyta) da Reserva Ecológica da Michelin, Igrapiúna, Bahia, Brasil. Sitientibus série Ciências Biológicas 8: 263-274.
Vilas Bôas-Bastos, S.B. \& Bastos, C.J.P. 2009. Musgos pleurocárpicos dos fragmentos de Mata Atlântica da Reserva Ecológica da Michelin, município de Igrapiúna, BA, Brasil. II - Hypnales (Bryophyta: Bryopsida). Acta Botanica Brasilica 23: 630-643.

Vilas Bôas-Bastos, S.B., Bastos, C.J.P. \& Costa, K.R. 2017. Brioflora da área de relevante interesse ecológico Serra do Orobó, municípios de Ruy Barbosa e Itaberaba, Bahia, Brasil. Pesquisas, Botânica 70: 79-98.

Yano, O. Briófitas. 1984. In: O. Fidalgo \& V.L.R. Bononi (eds.). Técnicas de coleta, conservação e herborização do material botânico. Instituto de Botânica, São Paulo, pp. 27-30.

Yano, O. 2011. Catálogo de musgos brasileiros: literatura original, basiônimo, localidade tipo e distribuição geográfica. 1 ed. Instituto de Botânica, São Paulo.

Yano, O. \& Peralta, D.F. 2006. Briófitas coletadas por Daniel Moreira Vital no Estado da Bahia. Boletim do Instituto de Botânica 18: 33-73. 